Archive for

Organic Chemistry

Arkivoc 2019, part iii, 8-21

\title{
Electrophilic acetylation and formylation of pyrrolo[1,2-a]pyrazines: substituent effects on regioselectivity
}

\author{
Dileep Kumar Singh and Ikyon Kim* \\ College of Pharmacy and Yonsei Institute of Pharmaceutical Sciences, Yonsei University \\ 85 Songdogwahak-ro, Yeonsu-gu, Incheon, 21983, Republic of Korea \\ Email: ikyonkim@yonsei.ac.kr
}

Dedicated to Professor George A. Kraus in celebration of his many outstanding contributions to organic synthesis

Received 11-07-2018

Accepted 12-16-2018

Published on line 01-12-2019

\section{Abstract}

Described herein is electrophilic acetylation and formylation of pyrrolo[1,2-a]pyrazines with substituent(s) at $\mathrm{C} 1$ and/or C3 position(s) where substituents around the core skeleton allowed regiodivergent access to C6- or C8-acetylpyrrolo[1,2-a]pyrazines. While acetylation of pyrrolo[1,2-a]pyrazines $(R(1)=H$ and $R(3)=$ aryl or methyl) mainly gave rise to C8-acetylated products, acetylation with pyrrolo[1,2-a]pyrazines $(R(1)=$ methyl and $\mathrm{R}(2)=$ aryl or methyl) provided C6-acetylated compounds as major products. In contrast, Vilsmeier-Haack formylation of pyrrolo[1,2-a]pyrazines took place at C6 position irrespective of the substituents at C1 and/or C3 site(s).

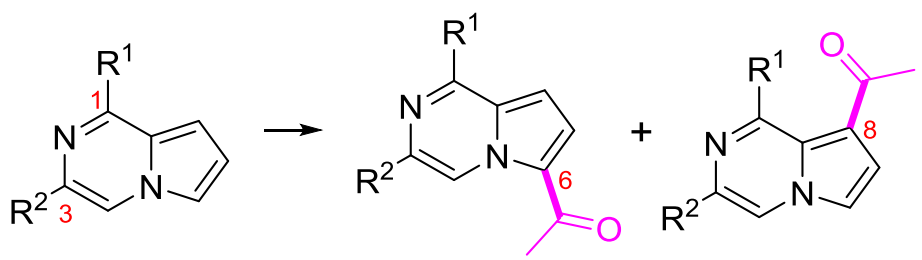

- Regiodivergent electrophilic acetylation

- Novel substitution patterns of pyrrolo[1,2-a]pyrazine

Keywords: Electrophilic acylation, pyrrolo[1,2-a]pyrazines, Vilsmeier-Haack formylation, Friedel-Crafts acylation; regioselectivity 


\section{Introduction}

Generation of novel heterocyclic chemical scaffolds, with unprecedented substitution patterns via regioselective installation of various functional groups around heterocyclic core skeletons, is highly important in the search for new effective pharmacophores in drug discovery programs. As illustrated in Figure 1, the bicyclic aza-heterocyclic pyrrolo[1,2-a]pyrazine system has been employed as a core chemical structure in a variety of medicinal chemistry efforts to discover an array of biological activities, including anticancer and antitubercular activities, depending on the substituents with different orientation. ${ }^{1-6}$

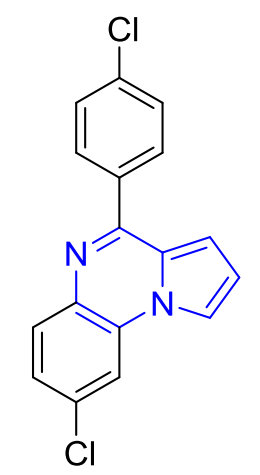

GPER anatagonist

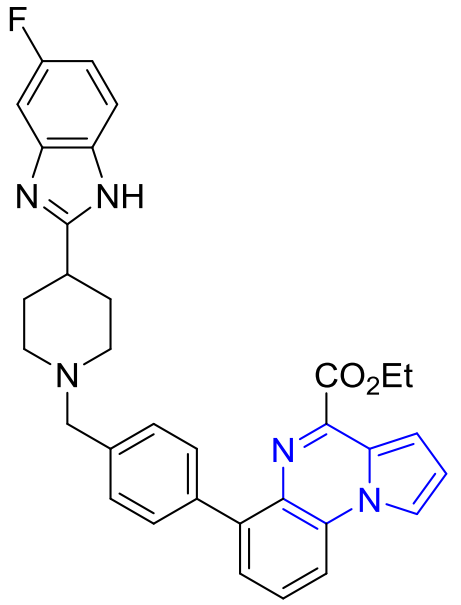

antileukemia agent<smiles>Cc1ccc(C(=O)C[n+]2cc3cccn3cc2-c2ccc(F)cc2)cc1</smiles>

Eis inhibitor (antitubercular agent)

Figure 1. Some bioactive pyrrolo[1,2-a]pyrazine derivatives

Clearly, these medicinal studies were made possible by synthetic strategies and technologies that enable a variety of functional groups to be arranged around the basic template at will. With the intention to construct a novel pyrrolo[1,2-a]pyrazine-based chemical library, we have reported on a diversity-oriented synthesis of pyrrolo[1,2-a]pyrazines with a (hetero)aryl moiety at the C6 position by way of Pd-catalyzed direct arylation (Scheme 1a). ${ }^{7}$ The resulting 6-arylpyrrolo[1,2-a]pyrazines were found to have anti-osteoporotic activity. ${ }^{8}$ To explore new territory based on pyrrolo[1,2-a]pyrazine, we investigated electrophilic acylation to install an acyl unit which could be further elaborated. From several literature precedents, ${ }^{9-15}$ we reasoned that Friedel-Crafts type acylation of $\mathbf{1}$ would mainly provide $\mathbf{3}$ having an acyl group at the C6 site (Scheme $1 \mathrm{~b}$ ).

Contrary to our expectation, we found that regioselectivity (C6 vs. C8) of acylation with 1 is highly dependent on the substituent(s) of 1 and the acylating agent, which is described herein. 
<smiles>[R]c1cn2cccc2c([R])n1</smiles>

1<smiles>[R4]c1cn2cccc2c([R])n1</smiles>

1

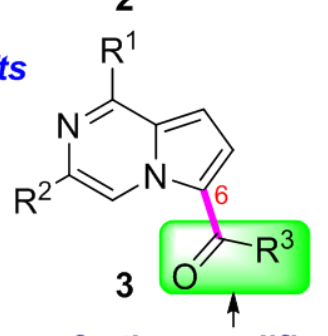

further modification (a)

Scheme 1. Synthetic-investigation plans: direct arylation using organometallic chemistry (1a) vs. acylation (1b).

\section{Results and Discussion}

As mentioned in the Introduction, we wished to introduce either an acetyl or a formyl group at the C6 position of $\mathbf{1}^{16}$ as a functional handle for further derivatization. For this purpose, we reacted the C3-phenyl compound 1a with acetyl chloride in the presence of $\mathrm{AlCl}_{3}$ as shown in Scheme 2. Two monoacetyl compounds were observed. NMR analysis revealed that the major product is the C8-acetyl pyrrolo[1,2-a]pyrazine (4a') (77\%) and the minor product is the C6-acetylated compound $\mathbf{4 a}$ (14\%). The structure of $\mathbf{4 a}$ ' was further confirmed by X-ray crystallographic analysis (Figure 2). ${ }^{17}$
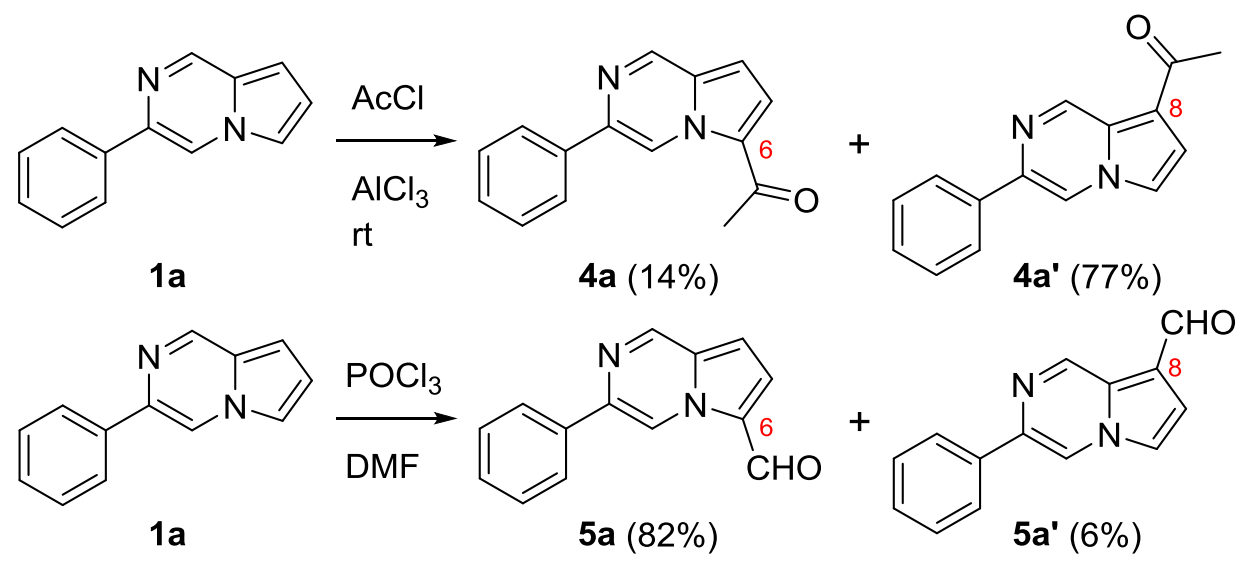<smiles>c1ccc(-c2cn3cccc3cn2)cc1</smiles><smiles>O=C(Cl)C1CCCCC1</smiles>
$\mathrm{rt}$

$1 \mathrm{a}$<smiles>O=C(c1ccccc1)c1ccn2cc(-c3ccccc3)ncc12</smiles>

Scheme 2. Electrophilic acylation of 1a. 


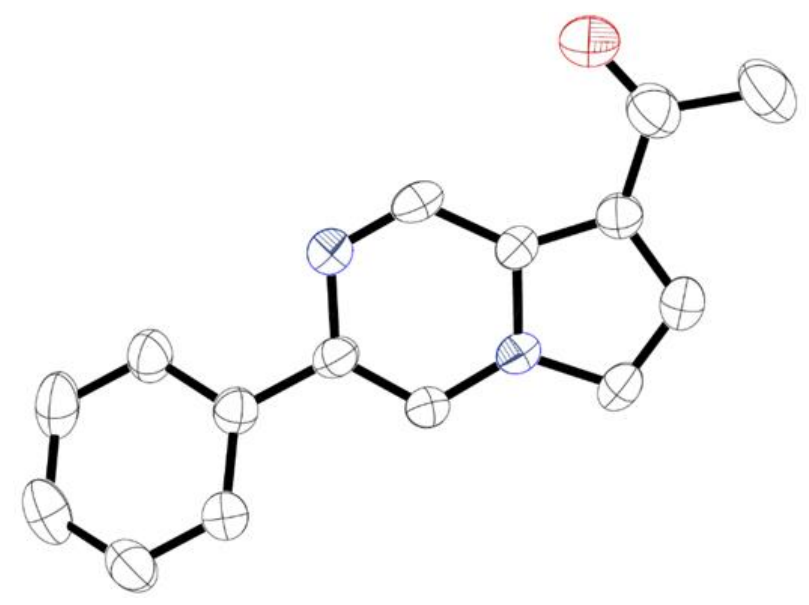

Figure 2. Crystal structure of 4a'.

This was quite surprising to us because several previous studies had indicated preferential acetylation at the C6 site of pyrrolo[1,2-a]pyrazines under similar conditions. On the other hand, Vilsmeier-Haack formylation $^{18}$ of 1 la produced the C6-formylated product (5a) as the major product. These two results led us to conclude that the steric effect of a phenyl moiety at C3 plays a crucial role in controlling the regioselectivity of acetylation. With our expectation that a bulkier group than acetyl would afford better C8 regioselectivity, we conducted benzoylation of $1 \mathbf{a}$ with $\mathrm{PhCOCl}$ and $\mathrm{AlCl}_{3}$, however, no selectivity was observed in this case. Rather, the C6-benzoylated compound (6a) was obtained slightly preferentially (46\% yield) to 6a' (33\%). These alterations of reaction sites in Friedel-Crafts type acylations, as well as a lack of systematic study with respect to substituent effects of pyrrolo[1,2-a]pyrazines on site-selective acylations, led us to investigate the roles of substituents in determining the regioselectivity of acylation.

To examine the general pattern of electrophilic acetylation and formylation, several pyrrolo[1,2-a]pyrazines were submitted to the identical conditions (Scheme 3 and Table 1). As expected, C8-acetylation occurred to afford 4' as the major products, irrespective of the electronic nature (electron-rich or poor) of the $\mathrm{R}^{2}$ moiety, whereas, products having a formyl group at $\mathrm{C} 6$ site were formed under Vilsmeier-Haack formylation conditions (entries 1-6). When $\mathrm{R}^{2}$ is methyl, a regioselective ratio of acetylation (C6 vs C8) decreases a little bit probably due to a smaller steric effect (entry 7). In cases of pyrrolo[1,2-a]pyrazines bearing a methyl at $R^{1}$ site (1f-h), C6-acetylation became a major pathway, indicating that the $\mathrm{R}^{1}$ substituent plays a bigger role than $\mathrm{R}^{2}$ in regioselection control (entries 9,11 , and 12 ).

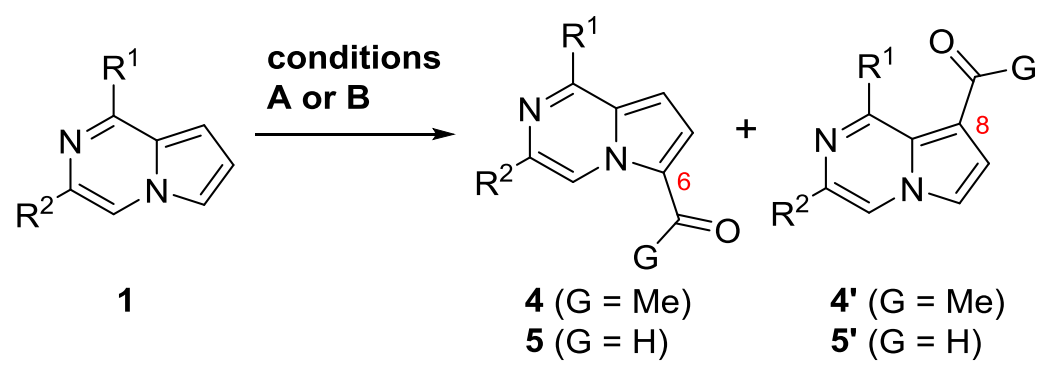

Scheme 3. Electrophilic acetylation and formylation reactions of pyrrolo[1,2-a]pyrazine (1). 
Table 1. Products of electrophilic acetylation and formylation reactions of $\mathbf{1}$

\begin{tabular}{|c|c|c|c|c|}
\hline Entry & 1 & Conditions $^{a}$ & & \\
\hline 1 & & A & $4 b(8)$ & $4 \mathbf{b}^{\prime}(73)$ \\
\hline 2 & & B & $5 \mathbf{b}(86)$ & $5 b^{\prime}(9)$ \\
\hline 3 & & A & $4 c(12)$ & $4 c^{\prime}(84)$ \\
\hline 4 & & B & $5 c(83)$ & $5 c^{\prime}(0)$ \\
\hline 5 & & A & $4 d(6)$ & 4d' (87) \\
\hline 6 & & B & 5d (95) & $5 d^{\prime}(0)$ \\
\hline 7 & & A & $4 e(13)$ & $4 e^{\prime}(66)$ \\
\hline 8 & $1 e$ & B & $5 e(80)$ & $5 e^{\prime}(14)$ \\
\hline 9 & & A & $4 f(67)$ & $4 f^{\prime}(6)$ \\
\hline 10 & & B & $5 f(65)$ & $5 f^{\prime}(0)$ \\
\hline 11 & & A & $4 g(77)$ & $4 \mathbf{g}^{\prime}(0)$ \\
\hline 12 & & A & $4 h(73)$ & $4 h^{\prime}(6)$ \\
\hline
\end{tabular}

${ }^{\text {a }}$ Conditions A: A mixture of 1 (30 mg), acetyl chloride (10 equiv), and $\mathrm{AlCl}_{3}$ (10 equiv) in $\mathrm{CH}_{2} \mathrm{Cl}_{2}\left(2.5 \mathrm{~mL}\right.$ ) was stirred at rt. Conditions $\mathrm{B}$ : To a mixture of $\mathrm{POCl}_{3}$ (5 equiv) in DMF (1 $\mathrm{mL}$ ) was added 1 (30 mg, dissolved in $0.5 \mathrm{~mL}$ of DMF) at rt. ${ }^{\mathrm{b}}$ Isolated yield (\%).

Further elaboration of the resulting acetyl- or formyl-containing pyrrolo[1,2-a]pyrazines was conducted (Scheme 4). 


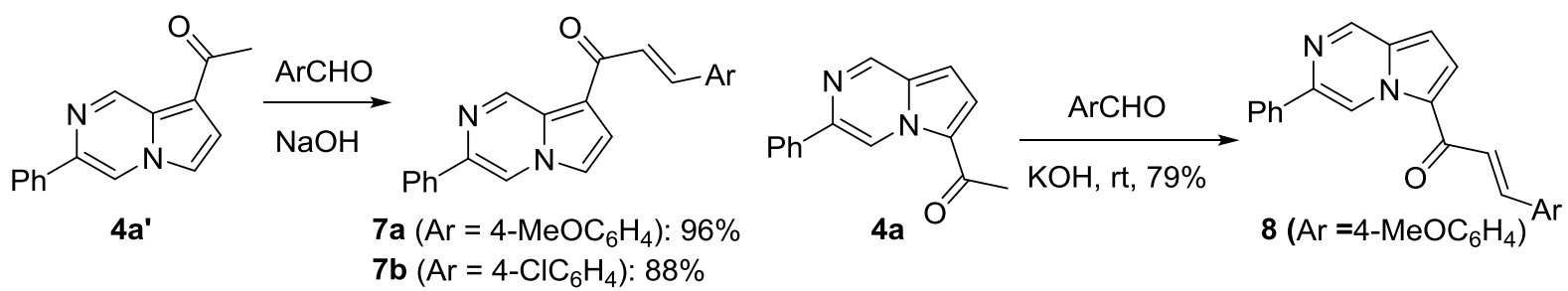<smiles>O=Cc1ccc2cnc(-c3ccccc3)cn12</smiles><smiles>COc1ccc(C(C)=O)cc1</smiles>

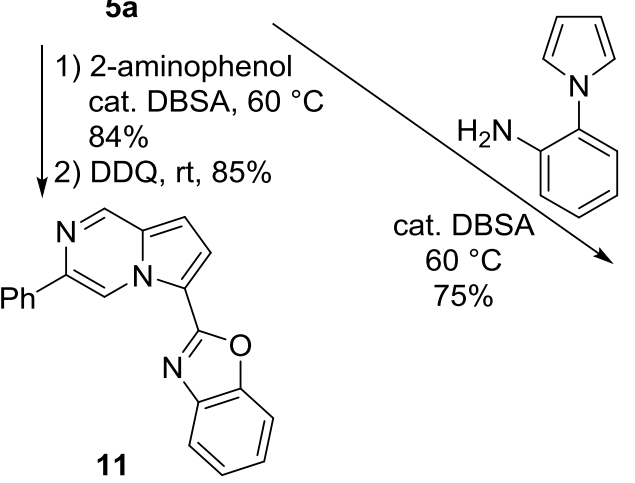<smiles>COc1ccc(C(=O)/C=C/c2ccc3cnc(-c4ccccc4)cn23)cc1</smiles><smiles></smiles>

Scheme 4. Further functionalization.

Base-mediated aldol condensation of $\mathbf{4} \mathbf{a}^{\prime}, \mathbf{4 a}$, and $\mathbf{5 a}$ proceeded well to give the corresponding chalcones $^{19-26}$ 7-9 in good yields. Notably, easy access to regioisomeric chalcones (7a, 8, and 9) was allowed by these acetylation/formylation-aldol procedures, enabling us to compare biological effects of these constitutional isomers bearing a chalcone moiety with different orientation. ${ }^{27}$ The formyl group in 5 a was also employed to install additional aromatic systems such as pyrrolo[1,2-a]quinoxaline ${ }^{28}$ and benzoxazole ${ }^{29}$ as shown in $\mathbf{1 0}$ and $\mathbf{1 1 .}$

\section{Conclusions}

In summary, we have investigated substituent effect on regioselectivity in electrophilic acetylation and formylation of pyrrolo[1,2-a]pyrazines. While Vilsmeier-Haack formylation proceeded mainly at the C6 position to give the corresponding C6-formyl products, Friedel-Crafts type acetylation occurred at the C6 or C8 positions as a major pathway depending on the pre-existing substituents of the substrates. New substitution patterns of pyrrolo[1,2-a]pyrazines were realized by these regiodivergent acylations. Further functionalization of this basic skeleton and biological tests of the synthesized compounds are currently in progress.

\section{Experimental Section}

General. Unless specified, all reagents and starting materials were purchased from commercial sources and used as received without purification. "Concentrated" refers to the removal of volatile solvents via distillation 
using a rotary evaporator. "Dried" refers to pouring onto, or passing through, anhydrous magnesium sulfate followed by filtration. Flash chromatography was performed using silica gel (230-400 mesh) with hexanes, ethyl acetate, and dichloromethane as the eluents. All reactions were monitored by thin-layer chromatography on $0.25 \mathrm{~mm}$ silica plates (F-254) visualized with UV light. Melting points were measured using a capillary melting point apparatus. ${ }^{1} \mathrm{H}$ and ${ }^{13} \mathrm{C}$ NMR spectra were recorded on a $400 \mathrm{MHz} \mathrm{NMR}$ spectrometer and were described as chemical shifts, multiplicity (s, singlet; $d$, doublet; $t$, triplet; $q$, quartet; m, multiplet), coupling constant in hertz $(\mathrm{Hz})$, and number of protons. HRMS was measured with an electrospray ionization (ESI) and Q-TOF mass analyzer.

General procedure A. To a stirred solution of the different pyrrolo[1,2-a]pyrazines (30 $\mathrm{mg}$ ) were added $\mathrm{AlCl}_{3}$ (10 equiv) and acetyl chloride (10 equiv) in $\mathrm{CH}_{2} \mathrm{Cl}_{2}(2.5 \mathrm{~mL})$ at room temperature. After being stirred at room temperature for 1-6 $\mathrm{h}$, the reaction mixture was poured into ice-cold water, neutralized with aqueous $\mathrm{NaHCO}_{3}$, and extracted with $\mathrm{CH}_{2} \mathrm{Cl}_{2}(5 \mathrm{~mL} \times 2)$. The organic layers were dried over $\mathrm{MgSO}_{4}$ and concentrated under reduced pressure to give the crude product. Purification by flash chromatography on silica gel (various hexanes:ethyl-acetate ratios) gave the corresponding products.

1-(3-Phenylpyrrolo[1,2-a]pyrazin-6-yl)ethanone (4a) and 1-(3-phenylpyrrolo[1,2-a]pyrazin-8-yl)ethanone (4a'). Prepared from 3-phenylpyrrolo[1,2-a]pyrazine (30 mg, $0.15 \mathrm{mmol}), \mathrm{AlCl}_{3}(200 \mathrm{mg}, 1.50 \mathrm{mmol})$, and acetyl chloride ( $107 \mu \mathrm{L}, 1.50 \mathrm{mmol}$ ) using general procedure $\mathbf{A}$ for $3 \mathrm{~h}$. Purification by flash chromatography on silica gel (hexanes:EtOAc, 9:1) afforded 4a (5 mg, 14\%) as a brown solid, mp 112.5-114.2 ${ }^{\circ} \mathrm{C} ;{ }^{1} \mathrm{H}$ NMR (400 $\left.\mathrm{MHz} \mathrm{CDCl}_{3}\right) \delta 10.04(\mathrm{~s}, 1 \mathrm{H}), 9.10(\mathrm{~s}, 1 \mathrm{H}), 8.01(\mathrm{~d}, J 7.2 \mathrm{~Hz}, 2 \mathrm{H}), 7.48-7.53(\mathrm{~m}, 3 \mathrm{H}), 7.40(\mathrm{t}, J 7.2 \mathrm{~Hz}, 1 \mathrm{H}), 6.82(\mathrm{~d}$, J $4.4 \mathrm{~Hz}, 1 \mathrm{H}), 2.63(\mathrm{~s}, 3 \mathrm{H}) ;{ }^{13} \mathrm{C} \mathrm{NMR}\left(100 \mathrm{MHz} \mathrm{CDCl}_{3}\right) \delta 188.7,144.2,141.2,137.1,131.1,128.9,128.7,126.5$, 124.4, 123.2, 116.8, 104.4, 27.8; HRMS (ESI-QTOF) $\mathrm{m} / \mathrm{z}[\mathrm{M}+\mathrm{H}]^{+}$calcd for $\mathrm{C}_{15} \mathrm{H}_{13} \mathrm{~N}_{2} \mathrm{O}$ 237.1022, found 237.1020. Purification by flash chromatography on silica gel (hexanes:EtOAc, 4:1) afforded 4a' (28 mg, 77\%) as a pale yellow solid; mp $172.5-174.2{ }^{\circ} \mathrm{C} ;{ }^{1} \mathrm{H}$ NMR $\left(400 \mathrm{MHz}_{\mathrm{CDCl}}\right) \delta 9.77(\mathrm{~s}, 1 \mathrm{H}), 8.28(\mathrm{~s}, 1 \mathrm{H}), 7.93(\mathrm{~d}, \mathrm{~J} 7.6 \mathrm{~Hz}, 2 \mathrm{H})$, 7.46-7.50 (m, 2H), 7.40-7.43 (m, 2H), $7.26(\mathrm{~s}, 1 \mathrm{H}), 2.59(\mathrm{~s}, 3 \mathrm{H}) ;{ }^{13} \mathrm{C} \mathrm{NMR}\left(100 \mathrm{MHz}, \mathrm{CDCl}_{3}\right) \delta 193.2,146.1,140.5$, $136.7,129.1,128.9,127.7,126.3,117.9,116.9,115.5,114.8,28.2 ;$ HRMS (ESI-QTOF) $\mathrm{m} / z$ [M+Na] ${ }^{+}$calcd for $\mathrm{C}_{15} \mathrm{H}_{12} \mathrm{~N}_{2} \mathrm{ONa} 259.0842$, found 259.0841 .

1-(3-(4-Methoxyphenyl)pyrrolo[1,2-a]pyrazin-6-yl)ethanone (4b) and 1-(3-(4-methoxyphenyl)-pyrrolo[1,2a]pyrazin-8-yl)ethanone (4b'). Prepared from 3-(4-methoxyphenyl)pyrrolo[1,2-a]pyrazine (30 mg, $0.13 \mathrm{mmol})$, $\mathrm{AlCl}_{3}(173 \mathrm{mg}, 1.30 \mathrm{mmol})$, and acetyl chloride $(92.4 \mu \mathrm{L}, 1.30 \mathrm{mmol}$ ) using general procedure $\mathrm{A}$ for $1 \mathrm{~h}$. Purification by flash chromatography on silica gel (hexanes:EtOAc, 9:1) afforded 4b (3 mg, 8\%) as a yellow solid, mp 144.4-146.2 ${ }^{\circ} \mathrm{C} ;{ }^{1} \mathrm{H}$ NMR $\left(400 \mathrm{MHz} \mathrm{CDCl}_{3}\right) \delta 9.97(\mathrm{~s}, 1 \mathrm{H}), 9.06(\mathrm{~s}, 1 \mathrm{H}), 7.95(\mathrm{~d}, J 8.4 \mathrm{~Hz}, 2 \mathrm{H}), 7.50(\mathrm{~d}, J$ $4.4 \mathrm{~Hz}, 1 \mathrm{H}), 7.02(\mathrm{~d}, J 8.4 \mathrm{~Hz}, 2 \mathrm{H}), 6.79(\mathrm{~d}, J 4.0 \mathrm{~Hz}, 1 \mathrm{H}), 3.87(\mathrm{~s}, 3 \mathrm{H}), 2.62(\mathrm{~s}, 3 \mathrm{H}) ;{ }^{13} \mathrm{C} \mathrm{NMR}\left(100 \mathrm{MHz}^{\mathrm{C}} \mathrm{CDCl}\right) \delta$ $188.7,160.3,144.1,141.2,130.9,129.7,127.8,124.3,123.1,115.9,114.4,104.3,55.5,27.9$; HRMS (ESI-QTOF) $\mathrm{m} / \mathrm{z}[\mathrm{M}+\mathrm{H}]^{+}$calcd for $\mathrm{C}_{16} \mathrm{H}_{15} \mathrm{~N}_{2} \mathrm{O}_{2}$ 267.1128, found 267.1123. Purification by flash chromatography on silica gel (hexanes:EtOAc, 4:1) afforded $\mathbf{4 \mathbf { b } ^ { \prime }}$ (26 mg, 73\%) as a brown solid, $\mathrm{mp}$ 206.2-208.1 ${ }^{\circ} \mathrm{C}$; ${ }^{1} \mathbf{H} \mathbf{~ N M R}(400 \mathrm{MHz}$, $\left.\mathrm{CDCl}_{3}\right) \delta 9.72(\mathrm{~s}, 1 \mathrm{H}), 8.18(\mathrm{~s}, 1 \mathrm{H}), 7.85(\mathrm{~d}, J 8.0 \mathrm{~Hz}, 2 \mathrm{H}), 7.35(\mathrm{~d}, J 2.4 \mathrm{~Hz}, 1 \mathrm{H}), 7.22(\mathrm{~d}, J 2.4 \mathrm{~Hz}, 1 \mathrm{H}), 7.99(\mathrm{~d}, J$ $8.0 \mathrm{~Hz}, 2 \mathrm{H}), 3.85(\mathrm{~s}, 3 \mathrm{H}), 2.57(\mathrm{~s}, 3 \mathrm{H}) ;{ }^{13} \mathrm{C}$ NMR $\left(100 \mathrm{MHz}, \mathrm{CDCl}_{3}\right) \delta 193.1,160.3,145.9,140.4,129.2,127.6$, 127.5, 117.8, 116.8, 115.3, 114.5, 113.8, 55.5, 28.1; HRMS (ESI-QTOF) $\mathrm{m} / z$ [M+H] ${ }^{+}$calcd for $\mathrm{C}_{16} \mathrm{H}_{15} \mathrm{~N}_{2} \mathrm{O}_{2}$ 267.1128, found 267.1125 .

1-(3-(4-Bromophenyl)pyrrolo[1,2-a]pyrazin-6-yl)ethanone (4c) and 1-(3-(4-bromophenyl)pyrrolo[1,2-a]pyrazin-8-yl)ethanone (4c'). Prepared from 3-(4-bromophenyl)pyrrolo[1,2-a]pyrazine (30 $\mathrm{mg}, 0.11 \mathrm{mmol})$, $\mathrm{AlCl}_{3}(147 \mathrm{mg}, 1.10 \mathrm{mmol})$, and acetyl chloride $(78.2 \mu \mathrm{L}, 1.10 \mathrm{mmol})$ using general procedure $\mathbf{A}$ for $1.5 \mathrm{~h}$. Purification by flash chromatography on silica gel (hexanes:EtOAc, $4: 1$ ) afforded $4 \mathrm{c}$ (4 mg, 12\%) as a pale 
yellow solid, mp 186.1-188.2 ${ }^{\circ} \mathrm{C} ;{ }^{1} \mathrm{H}$ NMR (400 MHz, CDCl $) \delta 10.01(\mathrm{~s}, 1 \mathrm{H}), 9.06(\mathrm{~s}, 1 \mathrm{H}), 7.88(\mathrm{~d}, J 8.4 \mathrm{~Hz}, 2 \mathrm{H})$, 7.60 (d, J $8.4 \mathrm{~Hz}, 2 \mathrm{H}), 7.52$ (d, J $4.4 \mathrm{~Hz}, 1 \mathrm{H}), 6.82$ (d, J $4.4 \mathrm{~Hz}, 1 \mathrm{H}), 2.62(\mathrm{~s}, 3 \mathrm{H}) ;{ }^{13} \mathrm{C} \mathrm{NMR}(100 \mathrm{MHz}, \mathrm{CDCl})_{3} \delta$ $188.9,144.3,139.9,136.0,132.1,131.0,127.9,124.5,123.4,123.0,116.8,104.6,27.9$; HRMS (ESI-QTOF) $m / z$ $[\mathrm{M}+\mathrm{H}]^{+}$calcd for $\mathrm{C}_{15} \mathrm{H}_{12} \mathrm{BrN}_{2} \mathrm{O} 315.0128$, found 315.0123. Purification by flash chromatography on silica gel $\left(\mathrm{CH}_{2} \mathrm{Cl}_{2}: \mathrm{MeOH}, 40: 1\right)$ afforded $4 \mathrm{c}^{\prime}(29 \mathrm{mg}, 84 \%)$ as an off-white solid, $\mathrm{mp}$ 226.6-228.2 ${ }^{\circ} \mathrm{C} ;{ }^{1} \mathrm{H} \mathrm{NMR}(400 \mathrm{MHz}$, $\left.\mathrm{CDCl}_{3}\right) \delta 9.74(\mathrm{~s}, 1 \mathrm{H}), 8.28(\mathrm{~s}, 1 \mathrm{H}), 7.81(\mathrm{~d}, J 8.4 \mathrm{~Hz}, 2 \mathrm{H}), 7.60(\mathrm{~d}, J 8.4 \mathrm{~Hz}, 2 \mathrm{H}), 7.40(\mathrm{~s}, 1 \mathrm{H}), 7.26(\mathrm{~s}, 1 \mathrm{H}), 2.59(\mathrm{~s}$, $3 \mathrm{H}) ;{ }^{13} \mathrm{C}$ NMR $\left(100 \mathrm{MHz} \mathrm{CDCl}_{3}\right) \delta 193.2,146.3,139.4,135.6,132.2,127.8,123.1,118.0,117.2,115.6,114.8$, 28.2; HRMS (ESI-QTOF) $m / z[\mathrm{M}+\mathrm{H}]^{+}$calcd for $\mathrm{C}_{15} \mathrm{H}_{12} \mathrm{BrN}_{2} \mathrm{O} 315.0128$, found 315.0125.

1-(3-(4-Chlorophenyl)pyrrolo[1,2-a]pyrazin-6-yl)ethanone (4d) and 1-(3-(4-chlorophenyl)pyrrolo[1,2a]pyrazin-8-yl)ethanone (4d'). Prepared from 3-(4-chlorophenyl)pyrrolo[1,2-a]pyrazine (30 mg, $0.13 \mathrm{mmol}$ ), $\mathrm{AlCl}_{3}(173 \mathrm{mg}, 1.30 \mathrm{mmol})$, and acetyl chloride $(92.4 \mu \mathrm{L}, 1.30 \mathrm{mmol})$ using general procedure $\mathbf{A}$ for $2 \mathrm{~h}$. Purification by flash chromatography on silica gel (hexanes:EtOAc, 9:1) afforded 4d (2 mg, 6\%) as a brown solid, mp 177.3-179.4 ${ }^{\circ} \mathrm{C} ;{ }^{1} \mathrm{H}$ NMR $\left(400 \mathrm{MHz} \mathrm{CDCl}_{3}\right) \delta 10.02(\mathrm{~s}, 1 \mathrm{H}), 9.08(\mathrm{~s}, 1 \mathrm{H}), 7.95(\mathrm{~d}, J 8.4 \mathrm{~Hz}, 2 \mathrm{H}), 7.53(\mathrm{~d}$, J $4.4 \mathrm{~Hz}, 1 \mathrm{H}), 7.45(\mathrm{~d}, J 8.4 \mathrm{~Hz}, 2 \mathrm{H}), 6.83(\mathrm{~d}, J 4.4 \mathrm{~Hz}, 1 \mathrm{H}), 2.63(\mathrm{~s}, 3 \mathrm{H}) ;{ }^{13} \mathrm{C} \mathrm{NMR}(100 \mathrm{MHz}, \mathrm{CDCl})_{3} \delta 188.9$, 144.3, 140.0, 135.6, 134.8, 131.0, 129.2, 127.7, 124.5, 123.4, 116.8, 104.6, 27.9; HRMS (ESI-QTOF) $\mathrm{m} / z$ [M+H] calcd for $\mathrm{C}_{15} \mathrm{H}_{12} \mathrm{ClN}_{2} \mathrm{O}$ 271.0633, found 271.0638. Purification by flash chromatography on silica gel (hexanes:EtOAc, 7:3) afforded 4d' (31 mg, 87\%) as a yellow solid, $\mathrm{mp} 214.2-216.4{ }^{\circ} \mathrm{C} ;{ }^{1} \mathrm{H} \mathrm{NMR}\left(400 \mathrm{MHz} \mathrm{CDCl}_{3}\right)$ $\delta 9.74(\mathrm{~d}, J 0.8 \mathrm{~Hz}, 1 \mathrm{H}), 8.26(\mathrm{~d}, J 1.2 \mathrm{~Hz}, 1 \mathrm{H}), 7.86(\mathrm{~d}, J 8.4 \mathrm{~Hz}, 2 \mathrm{H}), 7.43(\mathrm{~d}, J 8.4 \mathrm{~Hz}, 2 \mathrm{H}), 7.39(\mathrm{~d}, J 2.8 \mathrm{~Hz}$, $1 \mathrm{H}), 7.25-7.26(\mathrm{~m}, 1 \mathrm{H}), 2.59(\mathrm{~s}, 3 \mathrm{H}) ;{ }^{13} \mathrm{C} \mathrm{NMR}\left(100 \mathrm{MHz}, \mathrm{CDCl}_{3}\right) \delta 193.2,146.2,139.3,135.2,134.9,129.2$, 127.7, 127.5, 118.0, 117.1, 115.6, 114.8, 28.2; HRMS (ESI-QTOF) $\mathrm{m} / z[\mathrm{M}+\mathrm{H}]^{+}$calcd for $\mathrm{C}_{15} \mathrm{H}_{12} \mathrm{CIN}_{2} \mathrm{O} 271.0633$, found 271.0631.

1-(3-Methylpyrrolo[1,2-a]pyrazin-6-yl)ethanone (4e) and 1-(3-methylpyrrolo[1,2-a]pyrazin-8-yl)ethanone (4e'). Prepared from 3-methylpyrrolo[1,2-a]pyrazine (30 mg, $0.23 \mathrm{mmol}$ ), $\mathrm{AlCl}_{3}$ (307 $\mathrm{mg}, 2.30 \mathrm{mmol}$ ), and acetyl chloride $(163.5 \mu \mathrm{L}, 2.30 \mathrm{mmol})$ using general procedure $\mathbf{A}$ for $3 \mathrm{~h}$. Purification by flash chromatography on silica gel (hexanes:EtOAc, 9:1) afforded 4 e $(5.2 \mathrm{mg}, 13 \%)$ as a yellow solid, $\mathrm{mp} \mathrm{86.3-89.4}{ }^{\circ} \mathrm{C} ;{ }^{1} \mathrm{H}$ NMR (400 $\left.\mathrm{MHz} \mathrm{CDCl}_{3}\right) \delta 9.41(\mathrm{~s}, 1 \mathrm{H}), 8.94(\mathrm{~s}, 1 \mathrm{H}), 7.44(\mathrm{~d}, \mathrm{~J} 3.2 \mathrm{~Hz}, 1 \mathrm{H}), 6.74(\mathrm{~d}, J 4.0 \mathrm{~Hz}, 1 \mathrm{H}), 2.58(\mathrm{~s}, 3 \mathrm{H}), 2.53(\mathrm{~s}, 3 \mathrm{H})$;

${ }^{13} \mathrm{C} \mathrm{NMR}\left(100 \mathrm{MHz}, \mathrm{CDCl}_{3}\right) \delta 188.6,143.9,139.9,130.9,123.7,122.5,117.5,104.1,27.8,21.4$; HRMS (ESIQTOF) $m / z$ [M+Na] ${ }^{+}$calcd for $\mathrm{C}_{10} \mathrm{H}_{10} \mathrm{~N}_{2} \mathrm{ONa}$ 197.0685, found 197.0681. Purification by flash chromatography on silica gel (hexanes:EtOAc, 7:3) afforded $4 \mathrm{e}^{\prime}(26 \mathrm{mg}, 66 \%)$ as a pale yellow solid, $\mathrm{mp} 166.2-168.2{ }^{\circ} \mathrm{C} ;{ }^{1} \mathrm{H}$ NMR $\left(400 \mathrm{MHz}, \mathrm{CDCl}_{3}\right) \delta 9.60(\mathrm{~s}, 1 \mathrm{H}), 7.73(\mathrm{~s}, 1 \mathrm{H}), 7.24$ (br s, 1H), 7.17 (br s, 1H), $2.54(\mathrm{~s}, 3 \mathrm{H}), 2.46(\mathrm{~s}, 3 \mathrm{H}) ;{ }^{13} \mathrm{C} \mathrm{NMR}$ $\left(100 \mathrm{MHz}_{1} \mathrm{CDCl}_{3}\right) \delta 193.1,145.8,139.1,127.4,117.3,116.6,115.5,114.5,27.9,21.0$; HRMS (ESI-QTOF) $\mathrm{m} / \mathrm{z}$ $[\mathrm{M}+\mathrm{Na}]^{+}$calcd for $\mathrm{C}_{10} \mathrm{H}_{10} \mathrm{~N}_{2} \mathrm{ONa} 197.0685$, found 197.0685 .

1-(1-Methyl-3-phenylpyrrolo[1,2-a]pyrazin-6-yl)ethanone (4f) and 1-(1-methyl-3-phenylpyrrolo[1,2a]pyrazin-8-yl)ethanone (4f'). Prepared from 1-methyl-3-phenylpyrrolo[1,2-a]pyrazine (30 $\mathrm{mg}, 0.14 \mathrm{mmol}$ ), $\mathrm{AlCl}_{3}(187 \mathrm{mg}, 1.40 \mathrm{mmol})$, and acetyl chloride $(99.5 \mu \mathrm{L}, 1.40 \mathrm{mmol})$ using general procedure $\mathbf{A}$ for $6 \mathrm{~h}$. Purification by flash chromatography on silica gel (hexanes:EtOAc, 19:1) afforded $\mathbf{4 f}(24 \mathrm{mg}, 67 \%)$ as an orange solid, mp 157.6-159.8 ${ }^{\circ} \mathrm{C} ;{ }^{1} \mathrm{H}$ NMR $\left(400 \mathrm{MHz}, \mathrm{CDCl}_{3}\right) \delta 9.91(\mathrm{~s}, 1 \mathrm{H}), 8.01(\mathrm{~d}, J 7.2 \mathrm{~Hz}, 2 \mathrm{H}), 7.46-7.50(\mathrm{~m}, 3 \mathrm{H})$, 7.37-7.41 (m, 1H), $6.78(\mathrm{~d}, J 4.0 \mathrm{~Hz}, 1 \mathrm{H}), 2.83(\mathrm{~s}, 3 \mathrm{H}), 2.62(\mathrm{~s}, 3 \mathrm{H}) ;{ }^{13} \mathrm{C} \mathrm{NMR}\left(100 \mathrm{MHz}, \mathrm{CDCl}_{3}\right) \delta 188.7,152.5$, $140.7,137.4,130.7,128.9,128.5,126.6,124.8,122.8,115.2,103.8,27.9,22.2$; HRMS (ESI-QTOF) $\mathrm{m} / z$ [M+Na] $]^{+}$ calcd for $\mathrm{C}_{16} \mathrm{H}_{14} \mathrm{~N}_{2} \mathrm{ONa}$ 273.0998, found 273.0995. Purification by flash chromatography on silica gel (hexanes:EtOAc, 17:3) afforded $4 f^{\prime}(2.3 \mathrm{mg}, 6 \%)$ as an off-white solid, mp 123.6-125.8 ${ }^{\circ} \mathrm{C} ;{ }^{1} \mathrm{H} \mathrm{NMR}(400 \mathrm{MHz}$, $\left.\mathrm{CDCl}_{3}\right) \delta 8.13(\mathrm{~s}, 1 \mathrm{H}), 7.94(\mathrm{~d}, J 7.2 \mathrm{~Hz}, 2 \mathrm{H}), 7.45-7.49(\mathrm{~m}, 2 \mathrm{H}), 7.38-7.41(\mathrm{~m}, 2 \mathrm{H}), 7.26(\mathrm{~s}, 1 \mathrm{H}), 3.06(\mathrm{~s}, 3 \mathrm{H}), 2.63$ (s, 3H); ${ }^{13} \mathrm{C} N M R\left(100 \mathrm{MHz}, \mathrm{CDCl}_{3}\right) \delta 193.3,169.3,160.6,155.2,139.3,136.8,129.0,128.7,126.2,118.9,115.3$, 112.9, 297, 26.5; HRMS (ESI-QTOF) $\mathrm{m} / z$ [M+H] $]^{+}$calcd for $\mathrm{C}_{16} \mathrm{H}_{15} \mathrm{~N}_{2} \mathrm{O} 251.1179$, found 251.1177. 
1-[3-(4-Chlorophenyl)-1-methylpyrrolo[1,2-a]pyrazin-6-yl]ethanone (4g). Prepared from 3-(4-chlorophenyl)1-methylpyrrolo[1,2-a]pyrazine (30 mg, $0.12 \mathrm{mmol}), \mathrm{AlCl}_{3}(160 \mathrm{mg}, 1.20 \mathrm{mmol})$, and acetyl chloride $(85.3 \mu \mathrm{L}$, $1.20 \mathrm{mmol}$ ) using general procedure $\mathbf{A}$ for $2 \mathrm{~h}$. Purification by flash chromatography on silica gel (hexanes:EtOAc, 9:1) afforded $4 \mathrm{~g}(27 \mathrm{mg}, 77 \%)$ as a pale orange solid, mp 146.5-148.2 ${ }^{\circ} \mathrm{C} ;{ }^{1} \mathrm{H} \mathrm{NMR}(400 \mathrm{MHz}$, $\mathrm{CDCl}_{3}$ ) $\delta 9.89(\mathrm{~s}, 1 \mathrm{H}), 7.96(\mathrm{~d}, J 8.4 \mathrm{~Hz}, 2 \mathrm{H}), 7.49(\mathrm{~d}, J 4.8 \mathrm{~Hz}, 1 \mathrm{H}), 7.43(\mathrm{~d}, J 8.8 \mathrm{~Hz}, 2 \mathrm{H}), 6.78(\mathrm{~d}, J 4.4 \mathrm{~Hz}, 1 \mathrm{H})$, $2.81(\mathrm{~s}, 3 \mathrm{H}), 2.61(\mathrm{~s}, 3 \mathrm{H}) ;{ }^{13} \mathrm{C}$ NMR $\left(100 \mathrm{MHz} \mathrm{CDCl}_{3}\right) \delta 188.8,152.6,139.5,135.9,134.5,130.7,129.1,127.7$, 124.8, 122.9, 115.1, 103.9, 27.9, 22.1; HRMS (ESI-QTOF) $\mathrm{m} / \mathrm{z}[\mathrm{M}+\mathrm{H}]^{+}$calcd for $\mathrm{C}_{16} \mathrm{H}_{14} \mathrm{CIN}_{2} \mathrm{O} 285.0789$, found 285.0788.

1-(1,3-Dimethylpyrrolo[1,2-a]pyrazin-6-yl)ethanone $\quad(4 \mathrm{~h})$ and 1-(1,3-dimethylpyrrolo[1,2-a]pyrazin-8yl)ethanone (4h'). Prepared from 1,3-dimethylpyrrolo[1,2-a]pyrazine (30 mg, 0.21 mmol), AlCl 3 (280 mg, 2.10 $\mathrm{mmol})$, and acetyl chloride $(149.3 \mu \mathrm{L}, 2.10 \mathrm{mmol})$ using general procedure A for $1 \mathrm{~h}$. Purification by flash chromatography on silica gel (hexanes:EtOAc, 7:3) afforded $4 \mathrm{~h}(28.2 \mathrm{mg}, 73 \%)$ as a white solid, $\mathrm{mp} 120.2-$ $122.4{ }^{\circ} \mathrm{C} ;{ }^{1} \mathrm{H}$ NMR $\left(400 \mathrm{MHz}, \mathrm{CDCl}_{3}\right) \delta 9.30(\mathrm{~s}, 1 \mathrm{H}), 7.42(\mathrm{~d}, J 4.4 \mathrm{~Hz}, 1 \mathrm{H}), 6.72$ (d, J $\left.4.4 \mathrm{~Hz}, 1 \mathrm{H}\right), 2.73(\mathrm{~s}, 3 \mathrm{H})$, $2.58(\mathrm{~s}, 3 \mathrm{H}), 2.50(\mathrm{~s}, 3 \mathrm{H}) ;{ }^{13} \mathrm{C}$ NMR $\left(100 \mathrm{MHz} \mathrm{CDCl}_{3}\right) \delta 188.5,152.1,139.2,130.4,124.1,122.0,115.7,103.6$, 27.8, 21.9, 21.4; HRMS (ESI-QTOF) $\mathrm{m} / \mathrm{z}[\mathrm{M}+\mathrm{H}]^{+}$calcd for $\mathrm{C}_{11} \mathrm{H}_{13} \mathrm{~N}_{2} \mathrm{O}$ 189.1022, found 189.1023. Purification by flash chromatography on silica gel (hexanes:EtOAc, 3:2) afforded $4 \mathbf{h}^{\prime}$ (2.4 mg, 6\%) as a white solid, mp 157.4$159.6{ }^{\circ} \mathrm{C} ;{ }^{1} \mathrm{H}$ NMR $\left(400 \mathrm{MHz}, \mathrm{CDCl}_{3}\right) \delta 7.58(\mathrm{~s}, 1 \mathrm{H}), 7.23(\mathrm{~d}, J 2.8 \mathrm{~Hz}, 1 \mathrm{H}), 7.18(\mathrm{~d}, J 2.8 \mathrm{~Hz}, 1 \mathrm{H}), 2.96(\mathrm{~s}, 3 \mathrm{H})$, $2.60(\mathrm{~s}, 3 \mathrm{H}), 2.42$ (s, 3H); ${ }^{13} \mathrm{C}$ NMR $\left(100 \mathrm{MHz} \mathrm{CDCl}_{3}\right) \delta 193.3,154.9,137.8,127.5,125.7,118.4,114.3,113.7$, 29.6, 26.1, 20.9; HRMS (ESI-QTOF) $m / z[M+H]^{+}$calcd for $\mathrm{C}_{11} \mathrm{H}_{13} \mathrm{~N}_{2} \mathrm{O}$ 189.1022, found 189.1021 .

General procedure B. In a vial charged with DMF $(1.5 \mathrm{~mL})$ at $0{ }^{\circ} \mathrm{C}$ was added $\mathrm{POCl} 3$ (5 equiv) and the reaction mixture was stirred for $20 \mathrm{~min}$. To this mixture was added, dropwise, pyrrolo[1,2-a]pyrazine (30 mg, dissolved in $0.5 \mathrm{ml} \mathrm{DMF}$ ) at $0^{\circ} \mathrm{C}$. After being stirred at room temperature for $1-3 \mathrm{~h}$, the reaction mixture was poured into ice-cold water and neutralized with aqueous $\mathrm{NaHCO} 3$ solution. The reaction mixture was extracted with ethyl acetate $(5 \mathrm{~mL} \times 2)$, washed with aqueous $\mathrm{NaCl}(5 \mathrm{~mL} \times 2)$. The organic layers were dried over $\mathrm{MgSO} 4$ and concentrated under reduced pressure to give the crude product. Purification by flash chromatography on silica gel (hexanes:ethyl acetate) afforded the corresponding products.

3-Phenylpyrrolo[1,2-a]pyrazine-6-carbaldehyde (5a) and 3-phenylpyrrolo[1,2-a]pyrazine-8-carbaldehyde (5a'). Prepared from 3-phenylpyrrolo[1,2-a]pyrazine (30 mg, $0.15 \mathrm{mmol}$ ), and $\mathrm{POCl}_{3}(70 \mu \mathrm{L}, 0.75 \mathrm{mmol}$ ) using general procedure $\mathbf{B}$ for $3 \mathrm{~h}$. Purification by flash chromatography on silica gel (hexanes:EtOAc, 9:1) afforded $5 a(28.3 \mathrm{mg}, 82 \%)$ as a pale yellow solid, $\mathrm{mp} 95.6-97.8^{\circ} \mathrm{C} ;{ }^{1} \mathrm{H} \mathrm{NMR}\left(400 \mathrm{MHz}, \mathrm{CDCl}_{3}\right) \delta 9.93(\mathrm{~s}, 1 \mathrm{H}), 9.85(\mathrm{~s}, 1 \mathrm{H})$, $9.14(\mathrm{~s}, 1 \mathrm{H}), 8.01(\mathrm{~d}, J 8.0 \mathrm{~Hz}, 2 \mathrm{H}), 7.48-7.52(\mathrm{~m}, 3 \mathrm{H}), 7.42(\mathrm{t}, J 6.8 \mathrm{~Hz}, 1 \mathrm{H}), 6.86(\mathrm{~d}, J 4.4 \mathrm{~Hz}, 1 \mathrm{H}) ;{ }^{13} \mathrm{C} \mathrm{NMR}(100$ $\left.\mathrm{MHz}_{1} \mathrm{CDCl}_{3}\right) \delta 179.8,144.3,141.7,136.6,131.8,129.1,128.9,126.5,126.0,125.3,116.7,105.3$; HRMS (ESIQTOF) $m / z[\mathrm{M}+\mathrm{H}]^{+}$calcd for $\mathrm{C}_{14} \mathrm{H}_{11} \mathrm{~N}_{2} \mathrm{O} 223.0866$, found 223.0870. Purification by flash chromatography on silica gel (hexanes:EtOAc, 4:1) afforded 5a' $(2 \mathrm{mg}, 6 \%)$ as a brown solid, mp 144.4-146.2 ${ }^{\circ} \mathrm{C} ;{ }^{1} \mathrm{H} \mathrm{NMR}(400 \mathrm{MHz}$, $\left.\mathrm{CDCl}_{3}\right) \delta 10.13(\mathrm{~s}, 1 \mathrm{H}), 9.65(\mathrm{~s}, 1 \mathrm{H}), 8.34(\mathrm{~d}, J 1.2 \mathrm{~Hz}, 1 \mathrm{H}), 7.94(\mathrm{~d}, J 7.6 \mathrm{~Hz}, 2 \mathrm{H}), 7.41-7.52(\mathrm{~m}, 4 \mathrm{H}), 7.35(\mathrm{~d}, J$ $2.8 \mathrm{~Hz}, 1 \mathrm{H}) ;{ }^{13} \mathrm{C}$ NMR $\left(100 \mathrm{MHz} \mathrm{CDCl}_{3}\right) \delta 184.5,144.8,141.4,129.6,129.2,129.1,127.4,126.4,118.6,117.1$, 116.7, 115.2; HRMS (ESI-QTOF) $m / z[M+H]^{+}$calcd for $\mathrm{C}_{14} \mathrm{H}_{11} \mathrm{~N}_{2} \mathrm{O} 223.0866$, found 223.0864 .

3-(4-Methoxyphenyl)pyrrolo[1,2-a]pyrazine-6-carbaldehyde (5b) and 3-(4-Methoxyphenyl)pyrrolo[1,2a]pyrazine-8-carbaldehyde (56'). Prepared from 3-(4-methoxyphenyl)pyrrolo[1,2-a]pyrazine (30 mg, 0.13 $\mathrm{mmol}$ ) and $\mathrm{POCl}_{3}(60.7 \mu \mathrm{L}, 0.65 \mathrm{mmol})$ using general procedure $\mathbf{B}$ for $2 \mathrm{~h}$. Purification by flash chromatography on silica gel (hexanes:EtOAc, 9:1) afforded $5 \mathbf{b}(29 \mathrm{mg}, 86 \%)$ as a yellow solid, mp 132.5-134.2 ${ }^{\circ} \mathrm{C} ;{ }^{1} \mathrm{H}$ NMR (400 $\left.\mathrm{MHz}, \mathrm{CDCl}_{3}\right) \delta 9.91(\mathrm{~s}, 1 \mathrm{H}), 9.79(\mathrm{~s}, 1 \mathrm{H}), 9.11(\mathrm{~s}, 1 \mathrm{H}), 7.95(\mathrm{~d}, J 8.8 \mathrm{~Hz}, 2 \mathrm{H}), 7.48(\mathrm{~d}, J 4.8 \mathrm{~Hz}, 1 \mathrm{H}), 7.02(\mathrm{~d}, J 8.8$ $\mathrm{Hz}, 2 \mathrm{H}), 6.84$ (d, J $4.4 \mathrm{~Hz}, 1 \mathrm{H}), 3.88(\mathrm{~s}, 3 \mathrm{H}) ;{ }^{13} \mathrm{C} \mathrm{NMR}\left(100 \mathrm{MHz}, \mathrm{CDCl}_{3}\right) \delta 179.7,160.4,144.2,141.6,131.7$, 
129.2, 127.8, 125.8, 125.1, 115.7, 114.5, 105.1, 55.5; HRMS (ESI-QTOF) $m / z[M+H]^{+}$calcd for $\mathrm{C}_{15} \mathrm{H}_{13} \mathrm{~N}_{2} \mathrm{O}_{2}$ 253.0972, found 253.0979. Purification by flash chromatography on silica gel (hexanes:EtOAc, 7:3) afforded 5b' (3 mg, 9\%) as a brown solid, mp 170.2-172.6 ${ }^{\circ} \mathrm{C} ;{ }^{1} \mathrm{H} \mathrm{NMR}\left(400 \mathrm{MHz}, \mathrm{CDCl}_{3}\right) \delta 10.11(\mathrm{~s}, 1 \mathrm{H}), 9.62(\mathrm{~s}, 1 \mathrm{H}), 8.26$ (s, $1 \mathrm{H}), 7.88(\mathrm{~d}, J 8.8 \mathrm{~Hz}, 2 \mathrm{H}), 7.43(\mathrm{~d}, J 2.0 \mathrm{~Hz}, 1 \mathrm{H}), 7.33$ (d, J $2.4 \mathrm{~Hz}, 1 \mathrm{H}), 7.02$ (d, J $8.4 \mathrm{~Hz}, 2 \mathrm{H}), 3.87(\mathrm{~s}, 3 \mathrm{H})$; ${ }^{13} \mathrm{C} \mathrm{NMR}\left(100 \mathrm{MHz}, \mathrm{CDCl}_{3}\right) \delta 184.4,164.2,160.5,144.6,141.3,129.0,127.6,118.5,117.0,116.5,114.5,114.1$, 55.6; HRMS (ESI-QTOF) $\mathrm{m} / z[\mathrm{M}+\mathrm{H}]^{+}$calcd for $\mathrm{C}_{15} \mathrm{H}_{13} \mathrm{~N}_{2} \mathrm{O}_{2} 253.0972$, found 253.0974 .

3-(4-Bromophenyl)pyrrolo[1,2-a]pyrazine-6-carbaldehyde (5c). Prepared from 3-(4-bromophenyl)pyrrolo[1,2a]pyrazine (30 mg, $0.11 \mathrm{mmol}$ ) and $\mathrm{POCl}_{3}(51.4 \mu \mathrm{L}, 0.55 \mathrm{mmol})$ using general procedure $\mathbf{B}$ for $1 \mathrm{~h}$. Purification by flash chromatography on silica gel (hexanes:EtOAc, 19:1) afforded $\mathbf{5 c}(27.6 \mathrm{mg}, 83 \%)$ as a pale yellow solid, $\mathrm{mp}$ 132.6-135.2 ${ }^{\circ} \mathrm{C} ;{ }^{1} \mathrm{H}$ NMR $\left(400 \mathrm{MHz}, \mathrm{CDCl}_{3}\right) \delta 9.93(\mathrm{~s}, 1 \mathrm{H}), 9.85(\mathrm{~s}, 1 \mathrm{H}), 9.12(\mathrm{~s}, 1 \mathrm{H}), 7.89(\mathrm{~d}, J 8.4 \mathrm{~Hz}, 2 \mathrm{H})$, $7.61(\mathrm{~d}, J 8.4 \mathrm{~Hz}, 2 \mathrm{H}), 7.51$ (d, J $4.8 \mathrm{~Hz}, 1 \mathrm{H}), 6.87$ (d, J $4.8 \mathrm{~Hz}, 1 \mathrm{H}) ;{ }^{13} \mathrm{C} \mathrm{NMR}\left(100 \mathrm{MHz}, \mathrm{CDCl}_{3}\right) \delta$ 179.9, 144.5, $140.5,135.6,132.2,131.8,127.9,126.2,125.4,123.3,116.6,105.5 ; H R M S$ (ESI-QTOF) $\mathrm{m} / \mathrm{z}[\mathrm{M}+\mathrm{H}]^{+}$calcd for $\mathrm{C}_{14} \mathrm{H}_{10} \mathrm{BrN}_{2} \mathrm{O} 300.9971$, found 300.9967.

3-(4-Chlorophenyl)pyrrolo[1,2-a]pyrazine-6-carbaldehyde (5d). Prepared from 3-(4-chlorophenyl)pyrrolo[1,2a]pyrazine $(30 \mathrm{mg}, 0.13 \mathrm{mmol})$ and $\mathrm{POCl}_{3}(60.7 \mu \mathrm{L}, 0.65 \mathrm{mmol})$ using general procedure $\mathbf{B}$ for $1 \mathrm{~h}$. Purification by flash chromatography on silica gel (hexanes:EtOAc, 9:1) afforded $\mathbf{5 d}$ (32 $\mathrm{mg}, 95 \%)$ as a pale yellow solid, $\mathrm{mp}$ 123.9-125.2 ${ }^{\circ} \mathrm{C} ;{ }^{1} \mathrm{H}$ NMR $\left(400 \mathrm{MHz}, \mathrm{CDCl}_{3}\right) \delta 9.93(\mathrm{~s}, 1 \mathrm{H}), 9.83(\mathrm{~s}, 1 \mathrm{H}), 9.11(\mathrm{~s}, 1 \mathrm{H}), 7.95(\mathrm{~d}, J 8.4 \mathrm{~Hz}, 2 \mathrm{H}), 7.51$ (d, J $4.8 \mathrm{~Hz}, 1 \mathrm{H}), 7.46$ (d, J $8.4 \mathrm{~Hz}, 2 \mathrm{H}), 6.86$ (d, J $4.4 \mathrm{~Hz}, 1 \mathrm{H}) ;{ }^{13} \mathrm{C} N M R\left(100 \mathrm{MHz}, \mathrm{CDCl}_{3}\right) \delta 179.9,144.4,140.5$, $135.1,135.0,131.7,129.2,127.7,126.1,125.3,116.6,105.5 ;$ HRMS (ESI-QTOF) $\mathrm{m} / \mathrm{z}[\mathrm{M}+\mathrm{H}]^{+}$calcd for $\mathrm{C}_{14} \mathrm{H}_{10} \mathrm{CIN}_{2} \mathrm{O} 257.0476$, found 257.0480 .

3-Methylpyrrolo[1,2-a]pyrazine-6-carbaldehyde (5e) and 3-methylpyrrolo[1,2-a]pyrazine-8-carbaldehyde (5e'). Prepared from 3-methylpyrrolo[1,2-a]pyrazine (30 mg, $0.23 \mathrm{mmol})$ and $\mathrm{POCl}_{3}(107.5 \mu \mathrm{L}, 1.15 \mathrm{mmol})$ using general procedure $\mathbf{B}$ for $2 \mathrm{~h}$. Purification by flash chromatography on silica gel (hexanes:EtOAc, 9:1) afforded 5 e (29 mg, 80\%) as a pale yellow solid, $\mathrm{mp} 105.8-107.6{ }^{\circ} \mathrm{C} ;{ }^{1} \mathrm{H} \mathrm{NMR}\left(400 \mathrm{MHz}, \mathrm{CDCl}_{3}\right) \delta 9.85(\mathrm{~s}, 1 \mathrm{H})$, $9.25(\mathrm{~s}, 1 \mathrm{H}), 8.97(\mathrm{~s}, 1 \mathrm{H}), 7.42(\mathrm{~d}, J 4.8 \mathrm{~Hz}, 1 \mathrm{H}), 6.78(\mathrm{~d}, J 4.8 \mathrm{~Hz}, 1 \mathrm{H}), 2.54(\mathrm{~s}, 3 \mathrm{H}) ;{ }^{13} \mathrm{C} \mathrm{NMR}\left(100 \mathrm{MHz} \mathrm{CDCl}_{3}\right) \delta$ 179.6, 144.1, 140.6, 131.6, 125.3, 124.5, 117.5, 105.0, 21.2; HRMS (ESI-QTOF) $\mathrm{m} / z[\mathrm{M}+\mathrm{H}]^{+}$calcd for $\mathrm{C}_{9} \mathrm{H}_{9} \mathrm{~N}_{2} \mathrm{O}$ 161.0709, found 161.0715. Purification by flash chromatography on silica gel (hexanes:EtOAc, 4:1) afforded $5 \mathrm{e}^{\prime}(5 \mathrm{mg}, 14 \%)$ as a yellow solid, mp 114.2-116.6 ${ }^{\circ} \mathrm{C} ;{ }^{1} \mathrm{H} \mathrm{NMR}\left(400 \mathrm{MHz}, \mathrm{CDCl}_{3}\right) \delta 10.07(\mathrm{~s}, 1 \mathrm{H}), 9.50(\mathrm{~s}, 1 \mathrm{H})$, $7.79(\mathrm{~s}, 1 \mathrm{H}), 7.32(\mathrm{~d}, J 2.0 \mathrm{~Hz}, 1 \mathrm{H}), 7.28(\mathrm{~d}, J 2.0 \mathrm{~Hz}, 1 \mathrm{H}), 2.51(\mathrm{~s}, 3 \mathrm{H}) ;{ }^{13} \mathrm{C} \mathrm{NMR}\left(100 \mathrm{MHz}, \mathrm{CDCl}_{3}\right) \delta 184.5$, 144.6, 140.1, 127.7, 118.2, 116.9, 115.9, 115.8, 21.2; HRMS (ESI-QTOF) $\mathrm{m} / z[\mathrm{M}+\mathrm{H}]^{+}$calcd for $\mathrm{C}_{9} \mathrm{H}_{9} \mathrm{~N}_{2} \mathrm{O}$ 161.0709, found 161.0710 .

1-Methyl-3-phenylpyrrolo[1,2-a]pyrazine-6-carbaldehyde (5f). Prepared from 1-methyl-3-phenylpyrrolo[1,2a]pyrazine (30 mg, $0.14 \mathrm{mmol}$ ) and $\mathrm{POCl}_{3}(65.4 \mu \mathrm{L}, 0.70 \mathrm{mmol}$ ) using general procedure $\mathbf{B}$ for $2 \mathrm{~h}$. Purification by flash chromatography on silica gel (hexanes:EtOAc, 9:1) afforded $\mathbf{5 f}(22 \mathrm{mg}, 65 \%)$ as a yellow solid, $\mathrm{mp}$ 137.4-139.1 ${ }^{\circ} \mathrm{C} ;{ }^{1} \mathrm{H}$ NMR (400 MHz, $\left.\mathrm{CDCl}_{3}\right) \delta 9.90(\mathrm{~s}, 1 \mathrm{H}), 9.73(\mathrm{~s}, 1 \mathrm{H}), 8.01(\mathrm{~d}, J 8.0 \mathrm{~Hz}, 2 \mathrm{H}), 7.46-7.51(\mathrm{~m}, 3 \mathrm{H})$ 7.40 (t, J $7.2 \mathrm{~Hz}, 1 \mathrm{H}), 6.82$ (d, J $4.0 \mathrm{~Hz}, 1 \mathrm{H}), 2.85$ (s, 3H); ${ }^{13} \mathrm{C} \mathrm{NMR}\left(100 \mathrm{MHz}, \mathrm{CDCl}_{3}\right) \delta 179.8,152.7,141.2$, $136.9,131.5,128.9,128.8,126.5,125.6,115.0,104.7,22.2$; HRMS (ESI-QTOF) $\mathrm{m} / z[\mathrm{M}+\mathrm{H}]^{+}$calcd for $\mathrm{C}_{15} \mathrm{H}_{13} \mathrm{~N}_{2} \mathrm{O}$ 237.1022, found 237.1025 .

Phenyl-(3-phenylpyrrolo[1,2-a]pyrazin-6-yl)methanone (6a) and phenyl-(3-phenylpyrrolo[1,2-a]pyrazin-8yl)methanone (6a'). Prepared from 3-phenylpyrrolo[1,2-a]pyrazine (30 mg, 0.15 mmol), AlCl 3 (200 mg, 1.50 $\mathrm{mmol})$, and benzoyl chloride $(69.7 \mu \mathrm{L}, 0.60 \mathrm{mmol})$ using general procedure A for $5 \mathrm{~h}$. Purification by flash chromatography on silica gel (hexanes:EtOAc, 19:1) afforded 6a (21.2 mg, 46\%) as a yellow solid, mp 120.6$122.1{ }^{\circ} \mathrm{C} ;{ }^{1} \mathrm{H}$ NMR $\left(400 \mathrm{MHz}, \mathrm{CDCl}_{3}\right) \delta 10.13(\mathrm{~s}, 1 \mathrm{H}), 9.14(\mathrm{~s}, 1 \mathrm{H}), 8.05(\mathrm{~d}, J 8.0 \mathrm{~Hz}, 2 \mathrm{H}), 7.86(\mathrm{~d}, J 8.4 \mathrm{~Hz}, 2 \mathrm{H})$, $7.60(\mathrm{t}, J 7.2 \mathrm{~Hz}, 1 \mathrm{H}), 7.49-7.55(\mathrm{~m}, 4 \mathrm{H}), 7.40-7.44(\mathrm{~m}, 2 \mathrm{H}), 6.85(\mathrm{~d}, J 4.4 \mathrm{~Hz}, 1 \mathrm{H}) ;{ }^{13} \mathrm{C} \mathrm{NMR}\left(100 \mathrm{MHz} \mathrm{CDCl}_{3}\right) \delta$ 
186.2, 144.3, 141.3, 139.8, 137.1, 131.9, 131.7, 129.2, 129.0, 128.8, 128.6, 126.5, 126.0, 124.12, 117.0, 104.6; HRMS (ESI-QTOF) $\mathrm{m} / \mathrm{z}[\mathrm{M}+\mathrm{Na}]^{+}$calcd for $\mathrm{C}_{20} \mathrm{H}_{14} \mathrm{~N}_{2} \mathrm{ONa}$ 321.0998, found 321.0995. Purification by flash chromatography on silica gel (hexanes:EtOAc, 9:1) afforded 6a' (15.3 mg, 33\%) as a yellow solid, mp 183.6$185.2{ }^{\circ} \mathrm{C} ;{ }^{1} \mathrm{H}$ NMR $\left(400 \mathrm{MHz}, \mathrm{CDCl}_{3}\right) \delta 9.73(\mathrm{~s}, 1 \mathrm{H}), 8.32(\mathrm{~s}, 1 \mathrm{H}), 7.94(\mathrm{~d}, J 7.6 \mathrm{~Hz}, 2 \mathrm{H}), 7.88(\mathrm{~d}, J 6.8 \mathrm{~Hz}, 2 \mathrm{H})$, $7.58(\mathrm{t}, J 7.2 \mathrm{~Hz}, 1 \mathrm{H}), 7.47-7.53(\mathrm{~m}, 4 \mathrm{H}), 7.39-7.44(\mathrm{~m}, 2 \mathrm{H}), 7.21(\mathrm{~d}, J 2.8 \mathrm{~Hz}, 1 \mathrm{H}) ;{ }^{13} \mathrm{C} \mathrm{NMR}\left(100 \mathrm{MHz} \mathrm{CDCl}_{3}\right) \delta$ $190.3,146.1,140.7,139.9,136.7,131.8,129.2,129.1,128.9,128.5,126.3,119.7,115.8,115.6,114.9 ;$ HRMS (ESI-QTOF) $m / z[\mathrm{M}+\mathrm{H}]^{+}$calcd for $\mathrm{C}_{20} \mathrm{H}_{15} \mathrm{~N}_{2} \mathrm{O} 299.1179$, found 299.1174 .

(E)-3-(4-Methoxyphenyl)-1-(3-phenylpyrrolo[1,2-a]pyrazin-8-yl)prop-2-en-1-one (7a). To a stirred mixture of 1-(3-phenylpyrrolo[1,2-a]pyrazin-8-yl)ethan-1-one (4a) $(50 \mathrm{mg}, 0.21 \mathrm{mmol}$ ) and 4-methoxybenzaldehyde (26 $\mu \mathrm{L}, 0.21 \mathrm{mmol})$ in ethanol $(2 \mathrm{~mL})$ was added $\mathrm{NaOH}(42 \mathrm{mg}, 1.05 \mathrm{mmol})$ in water $(2 \mathrm{~mL})$ at room temperature. After being stirred at $60{ }^{\circ} \mathrm{C}$ for $16 \mathrm{~h}$, the reaction mixture was cooled to $\mathrm{rt}$ and the precipitated product was suction-filtered to give $7 \mathrm{a}$ as a yellow solid (72 mg, 96\%). mp 192.2-193.6 ${ }^{\circ} \mathrm{C} ;{ }^{1} \mathrm{H} \mathrm{NMR}\left(400 \mathrm{MHz}, \mathrm{CDCl}_{3}\right) \delta 9.92$ $(\mathrm{s}, 1 \mathrm{H}), 8.30(\mathrm{~s}, 1 \mathrm{H}), 7.94(\mathrm{~d}, J 7.2 \mathrm{~Hz}, 2 \mathrm{H}), 7.84(\mathrm{~d}, J 15.6 \mathrm{~Hz}, 1 \mathrm{H}), 7.62(\mathrm{~d}, J 8.8 \mathrm{~Hz}, 2 \mathrm{H}), 7.49(\mathrm{t}, J 7.2 \mathrm{~Hz}, 2 \mathrm{H})$, 7.44-7.36 (m, 4H), $6.94(\mathrm{~d}, J 8.4 \mathrm{~Hz}, 2 \mathrm{H}), 3.86(\mathrm{~s}, 3 \mathrm{H}) ;{ }^{13} \mathrm{C} \mathrm{NMR}\left(100 \mathrm{MHz}, \mathrm{CDCl}_{3}\right) \delta 184.2,161.5,146.6,142.4$, $140.7,136.7,130.2,129.1,128.9,128.7,128.0,126.3,121.2,117.9,117.1,115.6,115.0,114.5,55.5 ;$ HRMS (ESI-QTOF) $m / z[\mathrm{M}+\mathrm{H}]^{+}$calcd for $\mathrm{C}_{23} \mathrm{H}_{19} \mathrm{~N}_{2} \mathrm{O}_{2} 335.1441$, found 335.1445 .

(E)-3-(4-Chlorophenyl)-1-(3-phenylpyrrolo[1,2-a]pyrazin-8-yl)prop-2-en-1-one (7b). Prepared from 1-(3phenylpyrrolo[1,2-a]pyrazin-8-yl)ethan-1-one (4a) $(50 \mathrm{mg}, 0.21 \mathrm{mmol})$, 4-chlorobenzaldehyde $(30 \mathrm{mg}, 0.21$ $\mathrm{mmol})$, and $\mathrm{NaOH}(42 \mathrm{mg}, 1.05 \mathrm{mmol})$ in $\mathrm{EtOH} /$ water $(1: 1,4 \mathrm{~mL})$ using the same procedure as that for the synthesis of $7 \mathrm{a}$. Suction-filtration afforded $7 \mathrm{~b}$ as a yellow solid (66 mg, 88\%). mp 218.3-218.8 ${ }^{\circ} \mathrm{C} ;{ }^{1} \mathrm{H}$ NMR (400 $\mathrm{MHz}, \mathrm{CDCl}_{3}$ ) $\delta 9.91(\mathrm{~s}, 1 \mathrm{H}), 8.32$ (d, J $\left.1.6 \mathrm{~Hz}, 1 \mathrm{H}\right), 7.94$ (d, J $\left.7.2 \mathrm{~Hz}, 2 \mathrm{H}\right), 7.81$ (d, J $\left.15.6 \mathrm{~Hz}, 1 \mathrm{H}\right), 7.59$ (d, J 8.4 $\mathrm{Hz}, 2 \mathrm{H}), 7.50-7.38(\mathrm{~m}, 8 \mathrm{H}) ;{ }^{13} \mathrm{C} \mathrm{NMR}\left(100 \mathrm{MHz} \mathrm{CDCl}_{3}\right) \delta 183.6,161.1,146.3,140.9,136.5,136.0,133.6,129.5$, $129.2,128.9,128.8,128.6,126.2,123.7,117.3,117.0,115.6,114.9$; HRMS (ESI-QTOF) $\mathrm{m} / z$ [M+Na] ${ }^{+}$calcd for $\mathrm{C}_{22} \mathrm{H}_{15} \mathrm{ClN}_{2} \mathrm{NaO} 381.0765$, found 381.0767 .

(E)-3-(4-Methoxyphenyl)-1-(3-phenylpyrrolo[1,2-a]pyrazin-6-yl)prop-2-en-1-one (8). To a stirred solution of 1-(3-phenylpyrrolo[1,2-a]pyrazin-6-yl)ethanone (4a) $(50 \mathrm{mg}, 0.21 \mathrm{mmol})$ in ethanol $(3 \mathrm{~mL})$ was added $\mathrm{KOH}$ (71 $\mathrm{mg}, 1.26 \mathrm{mmol})$ in water $(0.5 \mathrm{~mL})$ at room temperature. After $10 \mathrm{~min}$, 4-methoxybenzaldehyde $(28 \mu \mathrm{L}, 0.23$ $\mathrm{mmol}$ ) was added to the reaction mixture. After being stirred at room temperature for $16 \mathrm{~h}$, the reaction mixture was poured into ice-cold water, neutralized with $1 \mathrm{M} \mathrm{HCl}$, and extracted with ethyl acetate $(5 \mathrm{~mL} \times 2)$. The organic layers were dried over $\mathrm{MgSO}_{4}$ and concentrated under reduced pressure to give the crude product. Purification by flash chromatography on silica gel (hexanes:ethyl acetate, 4:1) gave 8 as a yellow solid (59 mg, 79\%). mp 188.2-190.6 ${ }^{\circ} \mathrm{C} ;{ }^{1} \mathrm{H}$ NMR (400 MHz, $\left.\mathrm{CDCl}_{3}\right) \delta 10.27(\mathrm{~s}, 1 \mathrm{H}), 9.11(\mathrm{~s}, 1 \mathrm{H}), 8.05(\mathrm{~d}, J 7.6 \mathrm{~Hz}, 2 \mathrm{H})$, $7.86(\mathrm{~d}, J 15.6 \mathrm{~Hz}, 1 \mathrm{H}), 7.69$ (d, J $4.4 \mathrm{~Hz}, 1 \mathrm{H}), 7.63(\mathrm{~d}, J 8.4 \mathrm{~Hz}, 2 \mathrm{H}), 7.50$ (t, J 7.6 Hz, 2H), 7.39-7.43 (m, 2H), $6.96(d, J 8.4 \mathrm{~Hz}, 2 \mathrm{H}), 6.88(\mathrm{~d}, J 4.0 \mathrm{~Hz}, 1 \mathrm{H}), 3.87(\mathrm{~s}, 3 \mathrm{H}) ;{ }^{13} \mathrm{C} \mathrm{NMR}\left(100 \mathrm{MHz}, \mathrm{CDCl}_{3}\right) \delta 179.9,161.7,144.1$, 142.6, 141.1, 137.1, 131.4, 130.3, 129.0, 128.7, 127.7, 126.5, 125.7, 122.3, 120.8, 117.3, 114.5, 104.8, 55.6; HRMS (ESI-QTOF) $m / z[\mathrm{M}+\mathrm{H}]^{+}$calcd for $\mathrm{C}_{23} \mathrm{H}_{19} \mathrm{~N}_{2} \mathrm{O}_{2} 355.1441$, found 355.1442 .

(E)-1-(4-Methoxyphenyl)-3-(3-phenylpyrrolo[1,2-a]pyrazin-6-yl)prop-2-en-1-one (9). Prepared from 3phenylpyrrolo[1,2-a]pyrazine-6-carbaldehyde (5a) $(50 \mathrm{mg}, 0.22 \mathrm{mmol}), \mathrm{KOH}$ (74 mg, $1.32 \mathrm{mmol}$ ) in water (0.5 $\mathrm{mL})$, and 4-methoxyacetophenone $(36.3 \mathrm{mg}, 0.24 \mathrm{mmol})$ using the same procedure as that for the synthesis of 8. Purification by flash chromatography on silica gel (hexanes:EtOAc, 9:1) afforded 9 as a yellow solid (72 mg, 90\%). mp 193.4-195.8 ${ }^{\circ} \mathrm{C} ;{ }^{1} \mathrm{H}$ NMR (400 MHz, CDCl 3 ) $\delta 8.93$ (d, J $\left.0.8 \mathrm{~Hz}, 1 \mathrm{H}\right), 8.49(\mathrm{~s}, 1 \mathrm{H}), 8.22(\mathrm{~d}, J 15.2 \mathrm{~Hz}$, $1 \mathrm{H}), 8.08$ (d, J $8.8 \mathrm{~Hz}, 2 \mathrm{H}), 7.96(\mathrm{~d}, J 7.2 \mathrm{~Hz}, 2 \mathrm{H}), 7.57$ (d, J $15.2 \mathrm{~Hz}, 1 \mathrm{H}), 7.50(\mathrm{t}, J 7.2 \mathrm{~Hz}, 2 \mathrm{H}), 7.41-7.44(\mathrm{~m}$, $2 \mathrm{H}), 7.00(\mathrm{~d}, J 8.8 \mathrm{~Hz}, 2 \mathrm{H}), 6.91(\mathrm{~d}, J 4.4 \mathrm{~Hz}, 1 \mathrm{H}), 3.90(\mathrm{~s}, 3 \mathrm{H}) ;{ }^{13} \mathrm{C} N M R\left(100 \mathrm{MHz}, \mathrm{CDCl}_{3}\right) \delta 187.7,163.6,144.7$, 
139.9, 137.1, 131.3, 130.8, 130.6, 129.1, 128.8, 128.0, 126.3, 124.9, 118.6, 116.7, 114.0, 112.3, 106.3, 55.7; HRMS (ESI-QTOF) $m / z$ [M+Na] $]^{+}$calcd for $\mathrm{C}_{23} \mathrm{H}_{18} \mathrm{~N}_{2} \mathrm{O}_{2} \mathrm{Na} 377.1260$, found 377.1263.

4-(3-Phenylpyrrolo[1,2-a]pyrazin-6-yl)pyrrolo[1,2-a]quinoxaline (10). To a stirred solution of 5 a (50 mg, 0.22 $\mathrm{mmol})$ in THF $(2 \mathrm{~mL})$ were added 2-(1H-pyrrol-1-yl)aniline $(41.76 \mathrm{mg}, 0.26 \mathrm{mmol})$ and $p$ dodecylbenezenesulfonic acid (DBSA) $(14.36 \mathrm{mg}, 0.04 \mathrm{mmol})$. After being stirred at $60{ }^{\circ} \mathrm{C}$ for $16 \mathrm{~h}$, the reaction mixture was cooled to rt, diluted with ethyl acetate $(5 \mathrm{~mL})$, and washed with aqueous $\mathrm{NaHCO}_{3}(5 \mathrm{ml} \times 2)$ and water $(5 \mathrm{~mL})$. The aqueous layer was extracted with ethyl acetate $(5 \mathrm{~mL})$ one more time. The organic layers were dried over $\mathrm{MgSO}_{4}$ and concentrated under reduced pressure to give the crude product. Purification by flash chromatography on silica gel (hexanes:ethyl acetate, 9:1) gave 10 as a yellow solid (61 mg, 75\%). mp 170.7-173.8 ${ }^{\circ} \mathrm{C} ;{ }^{1} \mathrm{H}$ NMR $\left(400 \mathrm{MHz}, \mathrm{CDCl}_{3}\right) \delta 10.12(\mathrm{~s}, 1 \mathrm{H}), 9.06(\mathrm{~s}, 1 \mathrm{H}), 8.00-8.03(\mathrm{~m}, 4 \mathrm{H}), 7.91(\mathrm{~d}, J 8.0 \mathrm{~Hz}, 1 \mathrm{H})$, $7.74(\mathrm{~d}, J 4.4 \mathrm{~Hz}, 1 \mathrm{H}), 7.48-7.57(\mathrm{~m}, 4 \mathrm{H}), 7.39(\mathrm{t}, J 7.2 \mathrm{~Hz}, 1 \mathrm{H}), 7.24(\mathrm{~d}, J 4.0 \mathrm{~Hz}, 1 \mathrm{H}), 6.96-6.99(\mathrm{~m}, 2 \mathrm{H}) ;{ }^{13} \mathrm{C}$ NMR $\left(100 \mathrm{MHz}_{\mathrm{CDCl}}\right) \delta 145.9,144.5,138.8,138.0,135.6,130.2,129.7,128.9,128.2,127.6,126.9,126.3$, $125.6,124.7,123.1,119.8,116.2,115.0,114.3,113.9,108.2,104.5 ;$ HRMS (ESI-QTOF) $\mathrm{m} / z$ [M+H] ${ }^{+}$calcd for $\mathrm{C}_{24} \mathrm{H}_{17} \mathrm{~N}_{4} 361.1448$, found 361.1443 .

2-(3-Phenylpyrrolo[1,2-a]pyrazin-6-yl)benzoxazole (11). To a stirred solution of 5a (50 $\mathrm{mg}, 0.22 \mathrm{mmol}$ ) in ethanol ( $3 \mathrm{~mL}$ ) were added 2-aminophenol ( $48 \mathrm{mg}, 0.44 \mathrm{mmol}$ ) and p-dodecylbenezenesulfonic acid (7.18 $\mathrm{mg}$, $0.02 \mathrm{mmol})$. After being stirred at $60{ }^{\circ} \mathrm{C}$ for $16 \mathrm{~h}$, the reaction mixture was cooled to rt, diluted with ethyl acetate $(5 \mathrm{~mL})$, and washed with aqueous $\mathrm{NaHCO}_{3}(5 \mathrm{ml} \times 2)$ and water $(5 \mathrm{~mL})$. The aqueous layer was extracted with ethyl acetate $\left(5 \mathrm{~mL}\right.$ ) one more time. The organic layers were dried over $\mathrm{MgSO}_{4}$ and concentrated under reduced pressure to give the crude product. Purification by flash chromatography on silica gel (hexanes:ethyl acetate, 19:1) furnished the Schiff base product as a yellow solid (59 mg, 84\%). To this Schiff base product ( $59 \mathrm{mg}, 0.19 \mathrm{mmol}$ ) in $\mathrm{CH}_{2} \mathrm{Cl}_{2}(3 \mathrm{~mL}$ ) was added DDQ (65 mg, $0.29 \mathrm{mmol})$. After being stirred at room temperature for $2 \mathrm{~h}$, the reaction mixture was diluted with $\mathrm{CH}_{2} \mathrm{Cl}_{2}(5 \mathrm{~mL})$ and washed with aqueous $\mathrm{Na}_{2} \mathrm{CO}_{3}(5 \mathrm{~mL} \times 3)$ and water $(5 \mathrm{~mL} \times 2)$. The aqueous layer was extracted with ethyl acetate $(5 \mathrm{~mL})$ one $\mathrm{more}$ time. The organic layers were dried over $\mathrm{MgSO}_{4}$ and concentrated under reduced pressure to give the crude product. Purification by flash chromatography on silica gel (hexanes:ethyl acetate, 49:1) afforded $\mathbf{1 1}$ as a pale yellow solid (50 mg, 85\%). mp 206.2-208.6 ${ }^{\circ} \mathrm{C} ;{ }^{1} \mathrm{H}$ NMR (400 MHz, CDCl 3 ) $\delta 10.02(\mathrm{~s}, 1 \mathrm{H}), 9.08(\mathrm{~s}, 1 \mathrm{H}), 8.09(\mathrm{~d}, J$ $8.0 \mathrm{~Hz}, 2 \mathrm{H}), 7.82(\mathrm{~d}, J 7.2 \mathrm{~Hz}, 1 \mathrm{H}), 7.70(\mathrm{~d}, J 4.4 \mathrm{~Hz}, 1 \mathrm{H}), 7.53-7.60(\mathrm{~m}, 3 \mathrm{H}), 7.36-7.46(\mathrm{~m}, 3 \mathrm{H}), 6.96(\mathrm{~d}, J 4.0 \mathrm{~Hz}$, $1 \mathrm{H}) ;{ }^{13} \mathrm{C}$ NMR $\left(100 \mathrm{MHz} \mathrm{CDCl}_{3}\right) \delta 156.7,149.5,144.3,142.3,140.1,137.5,130.7,129.0,128.6,126.6,125.0$, 124.8, 119.8, 119.2, 116.1, 114.8, 110.4, 105.0; HRMS (ESI-QTOF) $\mathrm{m} / \mathrm{z}[\mathrm{M}+\mathrm{H}]^{+}$calcd for $\mathrm{C}_{20} \mathrm{H}_{14} \mathrm{~N}_{3} \mathrm{O} 312.1131$, found 312.1136 .

\section{Acknowledgements}

We thank the National Research Foundation of Korea (NRF-2017R1A2A2A05069364 and NRF2018R1A6A1A03023718) for generous financial support.

\section{Supplementary Material}

${ }^{1} \mathrm{H}$ and ${ }^{13} \mathrm{C}$ NMR spectra of synthesized compounds, and a CIF file for 4a'. Readers will be able to access supporting information using the link "Supplementary Material" in the journal issue contents page. 


\section{References and Notes}

1. Bhide, R. S.; Batt, D. G.; Cherney, R. J.; Cornelius, L. A. M.; Liu, Q.; Marcoux, D.; Neels, J.; Poss, M. A.; Qin, L.-Y.; Ruan, Z.; Shi, Q.; Srivastava, A. S.; Tino, J. A.; Watterson, S. H. WO 2016064957 A1 20160428.

2. Lee, Y. H.; Lee, J. M.; Kim, S. G.; Lee, Y. S. Bioorg. Med. Chem. 2016, 24, 2843. https://doi.org/10.1016/i.bmc.2016.04.054

3. Yarkova, M. A.; Mokrov, G. V.; Gudasheva, T. A.; Seredenin, S. B. Pharm. Chem. J. 2016, 50, 501. https://doi.org/10.1007/s11094-016-1476-0

4. Garzan, A.; Willby, M. J.; Ngo, H. X.; Gajadeera, C. S.; Green, K. D.; Holbrook, S. Y. L.; Hou, C.; Posey, J. E.; Tsodikov, O. V.; Garneau-Tsodikova, S. ACS Infect. Dis. 2017, 3, 302.

5. Desplat, V.; Vincenzi, M.; Lucas, R.; Moreau, S.; Savrimoutou, S.; Rubio, S.; Pinaud, N.; Bigat, D.; Enriquez, E.; Marchivie, M.; Routier, S.; Sonnet, P.; Rossi, F.; Ronga, L.; Guillon, J. ChemMedChem 2017, 12, 940. https://doi.org/10.1002/cmdc.201700049

6. Aiello, F.; Carullo, G.; Giordano, F.; Spina, E.; Nigro, A.; Garofalo, A.; Tassini, S.; Costantino, G.; Vincetti, P.; Bruno, A.; Radi, M. ChemMedChem 2017, 12, 1279.

https://doi.org/10.1002/cmdc.201700145

7. Park, S.; Jung, Y.; Kim, I. Tetrahedron 2014, 70, 7534.

https://doi.org/10.1016/j.tet.2014.08.003

8. Kim, I.; Kim, S. H. KR 2016006050 A 20160118.

9. Buchan, R.; Fraser, M.; Kong Thoo Lin, P. V. S. J. Org. Chem. 1989, 54, 1074. https://doi.org/10.1021/jo00266a015

10. Terenin, V. I.; Kabanova, E. V.; Tselischeva, N. A.; Ivanov, A. S.; Zyk, N. V. Chem. Heterocycl. Comp. 2007, 43, 1038.

\section{https://doi.org/10.1007/s10593-007-0162-2}

11. Terenin, V. I.; Butkevich, M. A.; Ivanov, A. S.; Tselischeva, N. A.; Kabanova, E. V. Chem. Heterocycl. Comp. 2008, 44, 73.

https://doi.org/10.1007/s10593-008-0010-z

12. Terenin, V. I.; Butkevich, M. A.; Ivanov, A. S.; Kabanova, E. V. Chem. Heterocycl. Comp. 2008, 44, 200. https://doi.org/10.1007/s10593-008-0026-4

13. Lee, J. H.; Kim, I. J. Org. Chem. 2013, 78, 1283.

https://doi.org/10.1021/jo302590a

14. Saini, H. K.; Kaswan, P.; Pericherla, K.; Kumar, A. Asian J. Org. Chem. 2015, 4, 1380.

https://doi.org/10.1002/ajoc.201500297

15. Frett, B.; McConnell, N.; Kharbanda, A.; Naresh, G.; Rounseville, B.; Warner, C.; Chang, J.; Debolske, N.; Li, H.-y. Tetrahedron 2018, 74, 4592.

https://doi.org/10.1016/i.tet.2018.07.027

16. For the synthesis of 1 , see ref 7 .

17. CCDC 1861591 contains the supplementary crystallographic data for compound 4a'. These data can be obtained free of charge from The Cambridge Crystallographic Data Centre via www.ccdc.cam.ac.uk/data request/cif

18. Jones, G.; Stanforth, S. P. Org. React. , 1997, 49, 1.

19. Singh, P.; Anand, A.; Kumar, V. Eur. J. Med. Chem. 2014, 85, 758.

https://doi.org/10.1016/j.ejmech.2014.08.033

20. Zhuang, C.; Zhang, W.; Sheng, C.; Zhang, W.; Xing, C.; Miao, Z. Chem. Rev. 2017, 117, 7762. 


\section{https://doi.org/10.1021/acs.chemrev.7b00020}

21. Gomes, M. N.; Muratov, E. N.; Pereira, M.; Peixoto, J. C.; Rosseto, L. P.; Cravo, P. V. L.; Andrade, C. H.; Neves, B. J. Molecules 2017, 22, 1210. https://doi.org/10.3390/molecules22081210

22. Qin, H.-L.; Leng, J.; Zhang, C.-P.; Jantan, I.; Amjad, M. W.; Sher, M.; Naeem-ul-Hassan, M.; Hussain, M. A.; Bukhari, S. N. A. J. Med. Chem. 2016, 59, 3549.

https://doi.org/10.1021/acs.jmedchem.6b00276

23. Zhang, J.; Yang, F.; Qiao, Z.; Zhu, M.; Zhou, H. Bioorg. Med. Chem. Lett. 2016, 26, 5797. https://doi.org/10.1016/j.bmcl.2016.10.024

24. Coskun, D.; Erkisa, M.; Ulukaya, E.; Coskun, M. F.; Ari, F. Eur. J. Med. Chem. 2017, 136, 212. https://doi.org/10.1016/j.ejmech.2017.05.017

25. Liu, Q.-H.; Wu, J.-J.; Li, F.; Cai, P.; Yang, X.-L.; Kong, L.-Y.; Wang, X.-B. Med. Chem. Commun. 2017, 8, 1459. https://doi.org/10.1039/C7MD00199A

26. Park, S.; Kim, E. H.; Kim, J.; Kim, S. H.; Kim, I. Eur. J. Med. Chem. 2018, 144, 435. https://doi.org/10.1016/i.ejmech.2017.12.056

27. Wempe, M. F.; Jutabha, P.; Quade, B.; Iwen, T. J.; Frick, M. M.; Ross, I. R.; Rice, P. J.; Anzai, N.; Endou, H. J. Med. Chem. 2011, 54, 2701. Biological studies of regioisomers as a result of regiodivergent acylation of benzofurans.

https://doi.org/10.1021/jm1015022

28. Singh, D. K.; Nath, M. Org. Biomol. Chem. 2015, 13, 1836.

https://doi.org/10.1039/C4OB02370F

29. Chang, J.; Zhao, K.; Pan, S. Tetrahedron Lett. 2002, 43, 951.

https://doi.org/10.1016/S0040-4039(01)02302-4 